\title{
La larve et la fourmi
}

\author{
Marie-Pascale Huglo
}

\begin{abstract}
It happens in the summer, in a half-empty train. Two women travel in the same compartment. Between the dreamer and the one who makes things happen, something «stirs». Their brief, soothing encounter wakes a tale that comes from far away.
\end{abstract}

Je marchais le long du train, c'était juillet, un dimanche. Je voyageais seule, sans valise. Il n'y avait pas foule sur le quai, certains se dépêchaient par principe mais personne ne courait. La ville s'était vidée pendant l'été. J'ai dépassé les premières classes et suis montée dans une voiture du milieu, la treize. Les voyageurs continuaient d'avancer, ils se dirigeaient vers la tête du train pour gagner quelques centaines de mètres à l'arrivée - des habitués du parcours, sûrement, qui savaient où monter, où descendre. J'avais composté mon billet «aller-retour dans la journée», je n'avais que ça à faire, aller là-bas la voir, rendez-vous à midi pile dans le hall, 1'accompagner au restaurant, l'aider à s'asseoir sur la banquette (elle préfère), boire, manger, raconter des histoires, rire avec elle et à quatre heures pile, l'embrasser, la quitter, reprendre le train en sens inverse, rentrer.

Dans la voiture numéro treize, j'étais sûre d'être tranquille. Le voyage rend superstitieux n'importe qui. Même si on ne croit pas vraiment au pouvoir de ces chiffres qui, dit-on, portent malheur, on pense quand même au pire, aux accidents-aux bombes-aux-vols, alors mieux vaut ne pas tenter le diable, et comme la treize est mal placée, trop loin de la bouche des gares, de toutes ces gares qui nous aspirent, on poursuit vers les voitures de tête, logique. J'étais seule dans le wagon. Me suis assise côté fenêtre, mon gros sac de toile sur le siège à ma droite. Une poudre blanche opacifiait la vitre, les voyageurs se hâtaient sur le quai comme à travers un brouillard sale. Un haut-parleur a annoncé le départ tout proche. Voix féminine bien sûr, avec cet accent suave qu'on voudrait entendre, ne serait-ce qu'une fois, se décomposer.

Des voyageurs entraient dans le compartiment par grappes, ils se jetaient sur un siège, installaient leurs affaires en remuant, le sifflet du départ a retenti, le train s'ébranlait. J'ai sorti un livre de mon sac de toile. De temps en temps, je jetais un coup d'œil par la fenêtre sans vraiment regarder, tags sur les murs, linge sur une corde, wagons abandonnés puis, de nouveau, mon livre. Un mal de cœur s'installait, léger, j'ai posé le bouquin sur mon sac, derrière la vitre s'ouvrait la campagne de juillet. Champs de blé moissonnés. Une petite route au sortir d'une ferme, des arbres, le soleil qui dardait et dehors, personne. Pas une voiture. Pas un souffle, à peine de l'ombre. Le défilement ininterrompu du paysage immobile me 
plongeait dans l'hébétude des journées chaudes des vacances d'été où, hypnotisée par le bourdonnement d'une mouche, je m'installais dans une torpeur douceâtre et, de l'après-midi entier, ne faisait rien. Le bruit d'un long déchirement m'a ramenée à la réalité du voyage. Un peu plus loin devant, dans la rangée opposée, une femme blonde munie d'une paire de ciseaux découpait un papier d'emballage qu'elle avait déroulé à l'envers sur sa tablette. Elle a remis le rouleau de papier et les ciseaux au fond de son cabas placé sur le siège à sa gauche, dont elle a sorti du scotch et un livre. Elle a disposé le livre bien au centre de sa tablette et s'est employée à l'emballer. Bruit de papier froissé: pas très fort, mais agaçant. Elle a finalement déposé son livre enveloppé dans son cabas, dehors défilaient une pâture vide, des pavillons bordés de terrasses grises, persiennes closes. Aux masses sombres ou claires dont les contours fondaient sous l'effet de la vitesse succédaient, comme suspendus dans le temps, un arbre au milieu d'un champ, un pommier dont les branches tordues glissaient avec douceur hors de mon champ de vision et me laissaient rêveuse, à vrai dire me berçaient.

Un second déchirement m'a arrachée à la torpeur. Au lieu des taches floues et des explosions de formes lentes, côté fenêtre, il y avait, côté couloir, la blonde affairée. Munie de la paire de ciseaux ressortie entre-temps de son gros cabas noir, elle coupait son papier d'emballage (le même), sortait un livre (un autre) et du scotch, prenait des mesures à deux doigts, pliait son papier avec bruit (recommençait plusieurs fois pour que l'emballage soit parfaitement ajusté), un bruit inégal, insistant, impossible à prévenir, qui forait notre compartiment, qui bousculait le calme relatif du wagon comme une toile claquant quelque part dans la nuit, comme une craie rayant le tableau, comme un pincement de ventre venu vous rappeler qu'il n'y aura pas de répit, pas de repos tant qu'elle, la blondinette, poursuivra l'enveloppement de ses momies de papier. Je la regardais aller du cabas à la tablette et l'imaginais déjà flanquée de deux ou trois mouflets qu'elle langerait, torcherait, rabibocherait et enverrait paître avec ce même soin de fourmi ingénieuse, ce même souci de l'enveloppe et du geste nets, cette indifférence absolue à ceux qui ne font pas partie de sa tribu, qui n'évoluent pas dans son monde quand bien même prendraient-ils le même train ou partageraient la même voiture, le même environnement sonore.

Elle s'est levée pour aller aux toilettes, robe d'été couleurs pastel, simple, seyante. Elle a bavardé un brin avec un homme qui lisait tranquillement. Il l'écoutait en souriant, elle causait penchée vers lui, passait les doigts dans ses cheveux, sa voix chuintait, elle retenait son rire, sortait frôlant mon siège. L'homme a repris sa lecture, le calme est revenu, j'ai tourné la tête vers la fenêtre blanchie, me suis laissée happer par les volumes étirés, les taches sombres, les éclats lumineux d'où se détachaient, parfois, le spectacle étrange d'une campagne fauchée sous le soleil. J'ai entrevu la blonde se rasseoir à sa place, farfouiller dans son cabas, le mal de cœur ne me lâchait pas, la blonde non plus; de nouveau, elle déchirait l'atmosphère - papier, ciseaux, cadeau... J'ai fermé les yeux mais le bruit des froissements me chiffonnait, je n'arrivais pas à penser à autre chose, son barouf plein la tête... Comme ça n'arrêtait pas, une rage rentrée me gagnait, une haine pour cette femme et toutes celles qu'elle rameutait à grand bruit, l'armée des 
fourmis industrieuses, des madones affairées qui polluent l'atmosphère, gâchent les vacances, pourrissent les voyages. J'ai rouvert les yeux, fixant sa nuque baissée, répétant mentalement arrête, arrête, arrête-toi! Ses mains n'arrêtaient pas, elles ressortaient un nouveau livre dès qu'elles avaient terminé un emballage, changeaient de papier cadeau, faisaient encore plus de ramdam. La tête chauve d'un voyageur assis juste devant elle s'est retournée d'un mouvement brusque. La blonde découpait un papier à motifs floraux, le froissait sans discontinuer, le chauve l'affrontait muettement, il l'aurait volontiers insultée qu'elle comprenne (le bruit que ça fait) mais rien, rien que ce regard assassin par-devant qui relayait le mien par-derrière et elle, indifférente aux ondes négatives qu'à coup sûr nous propagions, forte de son bon droit (elle avait payé sa place), plantée sur sa tablette avec ses ciseaux et son scotch, fignolait les coins d'un de ses livres à offrir (comme d'autres - parfois les mêmes - sont à jeter).

Jamais elle n'arrêterait! Elle avait de son côté les mères, les épouses, les compagnes, les amies, les collègues, les amantes, les sœurs, les complices, une armée de fourmis affairées qui ne perdaient pas une minute, turbinaient du matin au soir, du soir au matin, été comme hiver et l'inverse. Ça ne pouvait pas s'arrêter, des choses à faire il y en a toujours, les femmes le savent bien, elles le savent toutes, moi aussi je le sais, on n'en vient pas à bout de cette force-là, de cette organisation forcenée, féroce même, vorace. C'est le règne de l'ordre, de l'occupation, des cycles, de la cadence, des provisions, des prévisions, des consciences satisfaites mais sourdes, épanouies dans leur vacarme familier. Les femmes sont les premières à le seriner: ça n'arrête pas! La blonde persistait dans le labeur, je me demandais combien elle en avait encore, de livres à envelopper... Plus ça durait plus je désespérais (ç'aurait dû être le contraire), mais son cabas semblait sans fond et mon voyage, sans fin. Je ne pouvais ni lire ni vagabonder en pensée, elle m'écrasait, avec ses paquets, me clouait contre la vitre ni plus ni moins. Le chauve s'est retourné une deuxième fois, regard furibond, derrière la fenêtre des pavillons résidentiels défilaient. Je connaissais trop bien l'empire de cette femme, je faisais moi-même partie, par ailleurs, de l'armée des fourmis, qu'importe: je ne l'en détestais que plus et l'aurais volontiers foulée aux pieds, qu'on en finisse.

Est arrivé ce qui, dans les trains, rituellement arrive: le haut-parleur (voix masculine, dénuée bien entendu de toute suavité) annonçait l'entrée en gare de L., trois minutes d'arrêt, je répète, trois minutes d'arrêt. La blonde a replié son papier d'emballage, rangé ses outils et posé l'ultime cadeau sur le dessus de sa pile, petits lingots d'économie et d'adresse qu'elle distribuerait, assurément, tout à l'heure. Ne plus l'entendre déchirer l'air me donnait l'impression de plonger le visage dans une bassine d'eau glacée. Elle se tenait immobile, bien droite sur son siège, la main repliée sur la poignée de son cabas, de nouveau insignifiante, aussi anonyme qu'on peut l'être en voyage, balayée d'un coup, anéantie avant même d'avoir décampé, renvoyée dans son silence de fourmi, dans la nullité foncière de l'ouvrière désœuvrée. Par la fenêtre: quai, pancartes, des gens debout. Le train s'est arrêté. Elle est sortie la première, $\mathrm{j}$ 'ai regardé ailleurs. Une vieille attendait sur un banc, nous repartions déjà, la voix annonçait l'arrivée prochaine en gare de A., qui jouxtait celle de L.. Dehors des pavillons, des jardinets, des voitures garées 
sur le bas-côté, paysage étroit, temps désormais compté: trois minutes d'arrêt. Je suis descendue sans hâte, je marchais vers les escaliers, l'un à gauche (mécanique) l'autre à droite (de ciment). J'hésitais. L'escalier mécanique sortait de l'ombre, tube mince dont la bouche donnait en plein soleil. Un détritus ondulait sur le bord de la première marche roulante. Il tressautait sur place. Je restais un moment à le regarder se tordre dans des sursauts larvaires puis $j$ 'ai fait un pas, me suis laissée aspirer vers la ville d'en haut. Sur le Boulevard, dans la lumière poudreuse, c'était désert. Midi approchait. 